\title{
Variational Monte Carlo simulations using tensor-product projected states
}

\author{
Olga Sikora, ${ }^{1}$ Hsueh-Wen Chang, ${ }^{1}$ Chung-Pin Chou, ${ }^{2,}{ }^{*}$ Frank Pollmann, ${ }^{3, \dagger}$ and Ying-Jer Kao ${ }^{1, \ddagger}$ \\ ${ }^{1}$ Department of Physics, National Taiwan University, Taipei 1060\%, Taiwan \\ ${ }^{2}$ Beijing Computational Science Research Center, Beijing 100084, China \\ ${ }^{3}$ Max-Planck-Institut für Physik komplexer Systeme, 01187 Dresden, Germany
}

\begin{abstract}
We propose an efficient numerical method, which combines the advantages of recently developed tensor-network based methods and standard trial wave functions, to study the ground state properties of quantum many-body systems. In this approach, we apply a projector in the form of a tensor-product operator to an input wave function, such as a Jastrow-type or Hartree-Fock wave function, and optimize the tensor elements via variational Monte Carlo. The entanglement already contained in the input wave function can considerably reduce the bond dimensions compared to the regular tensor-product state representation. In particular, this allows us to also represent states that do not obey the area law of entanglement entropy. In addition, for fermionic systems, the fermion sign structure can be encoded in the input wave function. We show that the optimized states provide good approximations of the ground-state energy and correlation functions in the cases of two-dimensional bosonic and fermonic systems.
\end{abstract}

PACS numbers: $71.10 . \mathrm{Fd}, 02.70 . \mathrm{Ss}, 05.10 . \mathrm{Cc}$

\section{INTRODUCTION}

Interacting electron and spin models in two dimensions (2D) exhibit some of the most interesting phenomena in condensed-matter physics, e.g., superconductivity and topologically ordered spin-liquid phases. ${ }^{1,2}$ The simulation of these quantum many-body systems is, however, a big challenge for all available computation methods. For example, exact diagonalization (ED) allows only for very limited system sizes, the density matrix renormalization group (DMRG) ${ }^{3}$ is extremely efficient in onedimensional (1D) systems but becomes very challenging in higher dimensional systems, and quantum Monte Carlo (QMC) methods ${ }^{4}$ suffer from the infamous sign problem in fermionic and frustrated systems.

Over the past years, the class of tensor-product states has been shown to be a very promising tool. Most successful are matrix product states (MPS), ${ }^{5}$ which are the underlying base of the DMRG method, providing the most powerful tool to study 1D systems. Various generalization of the MPS approach to $2 \mathrm{D}$ have been proposed, including rather direct generalizations such as projected entangled pair states or tensor product states (TPS) ${ }^{6-11}$ as well as correlator product states or entangled-plaquette states. ${ }^{12-15}$ TPS-based algorithms have been successfully applied to various frustrated quantum spin systems and hard-core bosons in $2 \mathrm{D}^{16-19}$. Based on the concept of entanglement renormalization, the so-called tensor renormalization group (TRG) and multi scale entanglement renormalization group (MERA) methods have been developed and successfully applied to lattice systems. ${ }^{20-24}$ Yet, due to the scaling of computational complexity as a function of the dimension of the tensors (i.e., the bond dimension), the applicability of the algorithms is still limited. This limitation motivates a search for alternative tensor-network-based algorithms. Recently, variational Monte Carlo (VMC) methods have been proposed, improving a tensor-product state (TPS) by stochastically applying projectors which filter out excited states. ${ }^{25,26}$ In an approach closely related to our work, wave functions combining a correlator product state and a Slater determinant (or a Pfaffian) were studied. ${ }^{27,28}$ Hartree-Fock and configurational interaction based input wave functions were also used to achieve better accuracy and lower the required bond dimension in the DMRG procedure. ${ }^{29,30}$ These results suggest that it is important to choose the basis wave functions that already represent some key features of the physics of the system under investigation.

In this paper, we propose a method which combines $2 \mathrm{D}$ tensor product states with standard trial wave functions. Our study is a generalization of a recent work by Chou et al. ${ }^{31}$ in which matrix-product based projected wave functions were considered as trial wave functions for VMC simulations in a 1D model. The main idea is that we can use physical intuition to choose an input wave function that already contains some of the features of the ground state which are difficult to be captured by a TPS with small bond dimension. This way we can reduce the computational costs arising from the tensor contraction and still have a very good approximation of the ground-state wave function. Furthermore, using the VMC method to sample energies, we ensure that we approach the ground-state energy from above. We demonstrate the effectiveness of our approach by benchmarking the ground-state energies and correlations for several $2 \mathrm{D}$ interacting quantum systems, such as spinless fermionic and hardcore bosonic $t$ - $V$ models, as well as the spinful fermionic Hubbard model. In these quantum models, we find that a suitable Jastrow-type or Hartree-Fock wave function greatly helps the TPS to approach the ground state.

This paper is organized as follows. We begin by introducing the main concept of our approach and review some relevant TPS based methods in Sec. II. In Sec. III, we present our results and benchmarks for different bosonic 
(a)

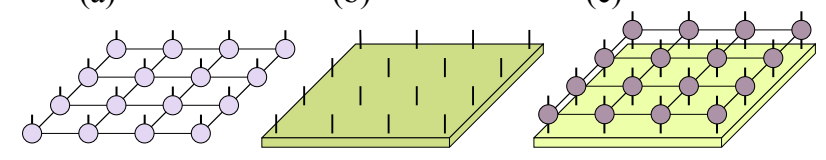

FIG. 1. Representations of (a) a TPS whose closed links represent bonds of dimension $D$ and open links physical indices; (b) an input $N$-particle wave function $\left|\Psi_{0}\right\rangle$ with indices depicted as open links; (c) a tensor-product projection operator acting on the wave function $\left|\Psi_{0}\right\rangle$ highlighted in green.

and fermionic models. We conclude with a summary and discussion in Sec. IV.

\section{METHODS}

\section{A. Tensor-network projected ansatz}

We consider tensor-network projected wave functions as a class of quantum states for Monte Carlo optimization, and in this section we discuss a general formula for such states. First, a TPS can be defined as

$$
\left|\Psi_{\mathrm{TPS}}\right\rangle=\sum_{j_{1}, \ldots, j_{N}} \mathrm{tTr}\left(\prod_{i=1}^{N} \hat{T}_{[i]}^{j_{i}}\right)|\alpha\rangle
$$

where $\alpha \equiv\left\{j_{1}, j_{2}, \cdots, j_{N}\right\}$ represents a many-body configuration in the lattice system of size $N, \hat{T}_{[i]}^{j_{i}}$ is the rank-4 tensor with bond dimension $D$, and the physical index $j_{i}$ representing a quantum state at site $i$. The configuration weight is given by contraction of a network of tensors as shown in Fig. 1(a), denoted as a tensorial trace tTr over all virtual bond indices. In this work we use this representation as one of the trial wave functions for simulating the bosonic system.

More generally, a representation of a quantum manybody state, which we call the tensor-product projected state (TPPS), can be written in terms of the projection operators acting on an input wave function $\left|\Psi_{0}\right\rangle$ :

$$
\left|\Psi_{\mathrm{TPPS}}\right\rangle=\sum_{j_{1}, \ldots, j_{N}} \mathrm{t} \operatorname{Tr}\left(\prod_{i=1}^{N} P_{[i]}^{j_{i}}\right)\left|\Psi_{0}\right\rangle,
$$

where $P_{[i]}^{j_{i}}=\hat{T}_{[i]}^{j_{i}}\left|j_{i}\right\rangle\left\langle j_{i}\right|$. If we assume $\left|\Psi_{0}\right\rangle=\sum_{\alpha}|\alpha\rangle$, i.e., a wave function with equal probability distribution of all configurations (a site-factorized state), we recover the TPS wave function of Eq. (1). However, we can also choose a different trial state, which might be closer to the ground state of a given system (e.g., incorporate entanglement) and thus require a smaller bond dimension of the tensor network. ${ }^{31} \mathrm{~A}$ schematic representation of the TPPS wave function is shown in Fig. 1(c). Expressed in a configuration basis, the TPPS is given by

$$
\left|\Psi_{\mathrm{TPPS}}\right\rangle=\sum_{j_{1}, \ldots, j_{N}} \mathrm{t} \operatorname{Tr}\left(\prod_{i=1}^{N} \hat{T}_{[i]}^{j_{i}}\right)\left\langle\alpha \mid \Psi_{0}\right\rangle|\alpha\rangle .
$$

In this work, we examine several different input states $\left|\Psi_{0}\right\rangle$ : (1) Jastrow wave function for hardcore bosons; (2) ground state wave function for noninteracting spinless fermions, i.e. Slater determinant (SL); (3) SL, $d$-wave BCS (d-BCS) and spin-density-wave (SDW) states for spinful fermions.

\section{B. Variational optimization of the wave function}

To optimize the ground-state energy, we apply standard quantum VMC methods ${ }^{32}$ to the TPPS wave function defined in Eq. (3). The expectation values of the observables are obtained as the average over the values for configurations visited during a Markov walk, and the probability distribution is given by coefficients of the wave function. The weight of the wave function for a given configuration $\alpha$ is

$$
W(\alpha)=\operatorname{tTr}\left(\prod_{i=1}^{N} \hat{T}_{[i]}^{j_{i}}\right)\left\langle\alpha \mid \Psi_{0}\right\rangle,
$$

where the tensorial trace corresponds to the contraction of a single-layer tensor network with $N$ open indices given by $\alpha$. The energy, for given tensors $\hat{T}_{[i]}^{j_{i}}$, can be written in the form appropriate for Monte Carlo sampling,

$$
\begin{aligned}
& E=\langle E(\alpha)\rangle=\frac{1}{Z} \sum_{\alpha} W^{2}(\alpha) E(\alpha), \\
& Z=\sum_{\alpha} W^{2}(\alpha),
\end{aligned}
$$

where the estimator is given by

$$
E(\alpha)=\sum_{\alpha^{\prime}} \frac{W\left(\alpha^{\prime}\right)}{W(\alpha)}\left\langle\alpha^{\prime}|\hat{H}| \alpha\right\rangle .
$$

The energy can be evaluated using importance sampling of the configurations according to the weight $W^{2}(\alpha)$.

We employ the stochastic reconfiguration (SR) method $^{32}$ to handle the multi-variable optimization of a wave function in the part of the Hilbert space spanned by its degrees of freedom. The SR method is a projector based optimization using the Hamiltonian to filter out the lowest energy state within the available subspace of the Hilbert space. The trial wave function can be also expanded in the variational parameters, which leads to a system of linear equations, with a new set of parameters as solutions. For the TPPS wave function, both the elements of tensors and parameters of the trial wave function might be variationally optimized. More details about the SR method can be found in Refs. 33 and 34 . The Monte Carlo sampling scheme ensures that the wave 
function is variational, and the energy fulfills the variational principle, i.e., the approximated energy provides an upper bound for the exact energy. In contrast, this is not necessarily the case when the wave function is obtained using the simplified update and the tensor network is contracted approximately. ${ }^{22}$

\section{Contraction of the tensor network}

During the simulation, we need to evaluate the contribution from the tensor network to the weight. We perform exact tensor contraction for $4 \times 4$ systems with bond dimension $D=2$ in the $t-V$ model and for all bond dimensions considered in the study of the Hubbard model. To efficiently evaluate the tensor network weight beyond this system size and/or bond dimension, we adopt the coarse-graining TRG method tailored for the Monte Carlo sampling. ${ }^{19,21,22}$ We briefly review the TRG procedure for contracting a $2^{n} \times 2^{n}$ tensor network, and illustrate it schematically in Fig. 2(a)-(d) for a $4 \times 4$ system. The tensor contraction is done by coarsegraining the lattice until the $2 \times 2$ tensor network can be directly contracted. In each step, we perform a singular value decomposition (SVD) to decompose a rank-4 tensor on each site of the lattice into two rank-3 tensors, keeping only the largest $D_{f}$ singular values, as shown in Fig. 2(b). Figures 2(c) and (d) show how the tensors are contracted to form a smaller lattice, for which the procedure is repeated. In the current simulation, the convergence can be reached by setting the cutoff $D_{f}$ to very small values of 2 or 3, retaining relatively low computational costs. In general, however, the cost of contracting a tensor network with a given cutoff $D_{f}$ grows rapidly with $D$, e.g. it takes almost 20 times longer to contract a $D=3$ than a $D=2$ TPS with $D_{f}=8$. It is thus important to start with a good trial wave function rather than to blindly increase the bond dimension of the tensors.

During the simulation, we apply local updates and change the physical state accordingly. We can lower the computational cost of tensor contraction by using part of the results from the previous step and updating only the elements that are changed at each stage of the coarse graining procedure. ${ }^{19}$ This way of updating elements is particularly helpful for larger lattice systems. In the following section, we illustrate the concept of the TPPS wave function using several examples.

\section{NUMERICAL RESULTS}

\section{A. Hardcore bosons}

We first discuss the hardcore boson model with nearest-neighbor repulsion (the $t-V$ model) at half filling on the square lattice. The motivation for this study is to test if the TPPS approach with suitable input wave (a)

(c)
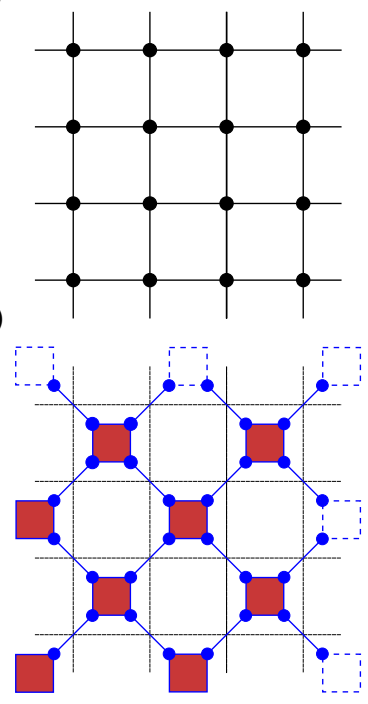

(b)

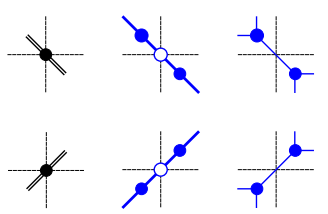

(d)

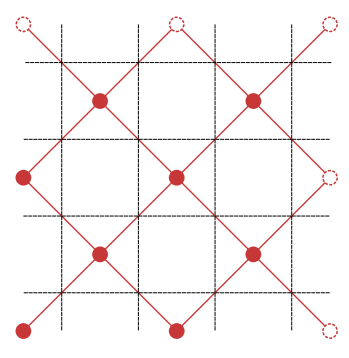

FIG. 2. TRG algorithm: (a) Bipartite network of sixteen tensors with periodic boundary conditions, where the tensor elements depend on the value of the physical index (site occupation) and the sublattice index. (b) Singular value decomposition and truncation at $D_{f}$, the middle matrix represented by empty circle is then absorbed into tensors (filled circles). (c) Contracting the tensors in the centers of the plaquettes yields a network of eight tensors (d).

functions can significantly improve the ground-state energy and correlations compared to TPS.

The Hamiltonian of this model is given by

$$
H_{t V}=-t \sum_{\langle i j\rangle}\left(b_{i}^{\dagger} b_{j}+H . c .\right)+V \sum_{\langle i j\rangle} n_{i} n_{j},
$$

where $t=1$ is the hopping integral, $V$ denotes the repulsion strength, $b_{i}^{(\dagger)}$ annihilates (creates) a hardcore boson on site $i$, and $n_{i}=b_{i}^{\dagger} b_{i}$. The hardcore boson can be mapped to the spin- $1 / 2$ antiferromagnetic (AF) XXZ model, with half filling corresponding to the zero magnetization sector. ${ }^{35}$ The hopping terms translate to the spin flipping, while the interaction terms map to the Ising interactions. The point $V=2 t$ corresponds to the Heisenberg point of the XXZ model, and the XY-Ising change in universality class corresponds to the transition between a superfluid phase and a charge-density-wave (CDW) insulator at $V=2 t$.

We simulate the model Hamiltonian Eq. (8) by examining several input wave functions including the Jastrowtype wave function, the TPS and the TPPS with a Jastrow input wave function. The simplest variational wave function we consider for this model is a Jastrow-type wave function $\left|\Psi_{\text {Jastrow }}\right\rangle$ with two variational parameters $(\gamma$ and $\beta$ ) associated with the local particle-particle correlations. The weight of a given configuration $\alpha$ is given by

$$
\left\langle\alpha \mid \Psi_{\text {Jastrow }}\right\rangle=\left\langle\alpha\left|\hat{J}_{\gamma, \beta}\right| \alpha\right\rangle .
$$


$(a)$

(b)
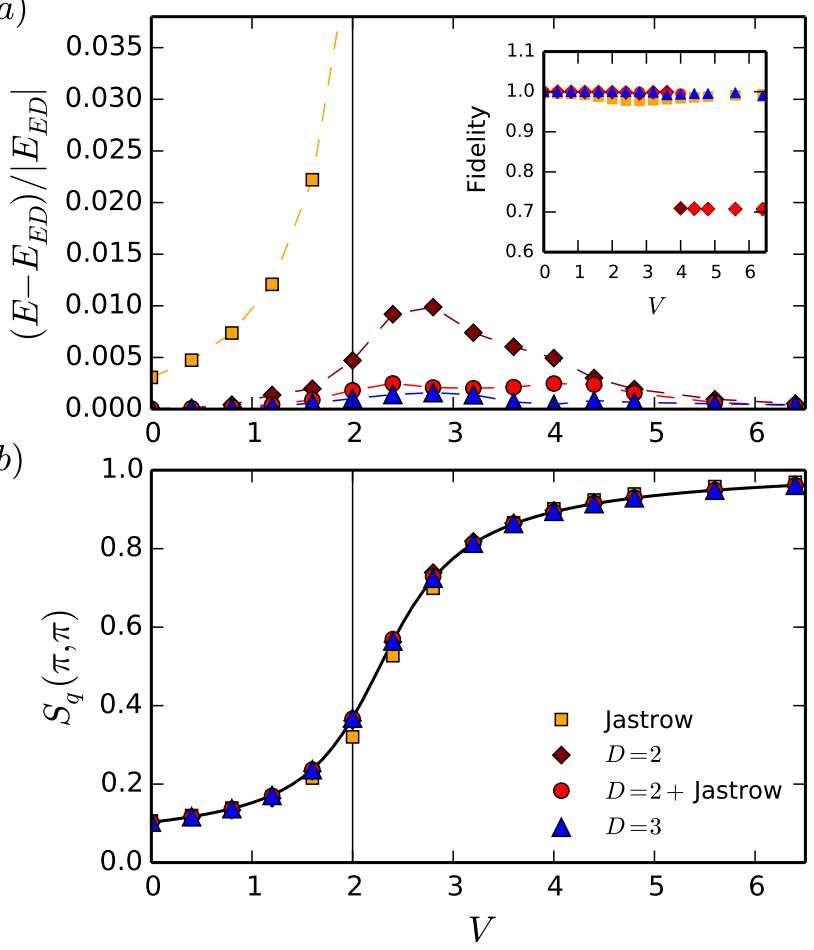

$(c)$

$(d)$
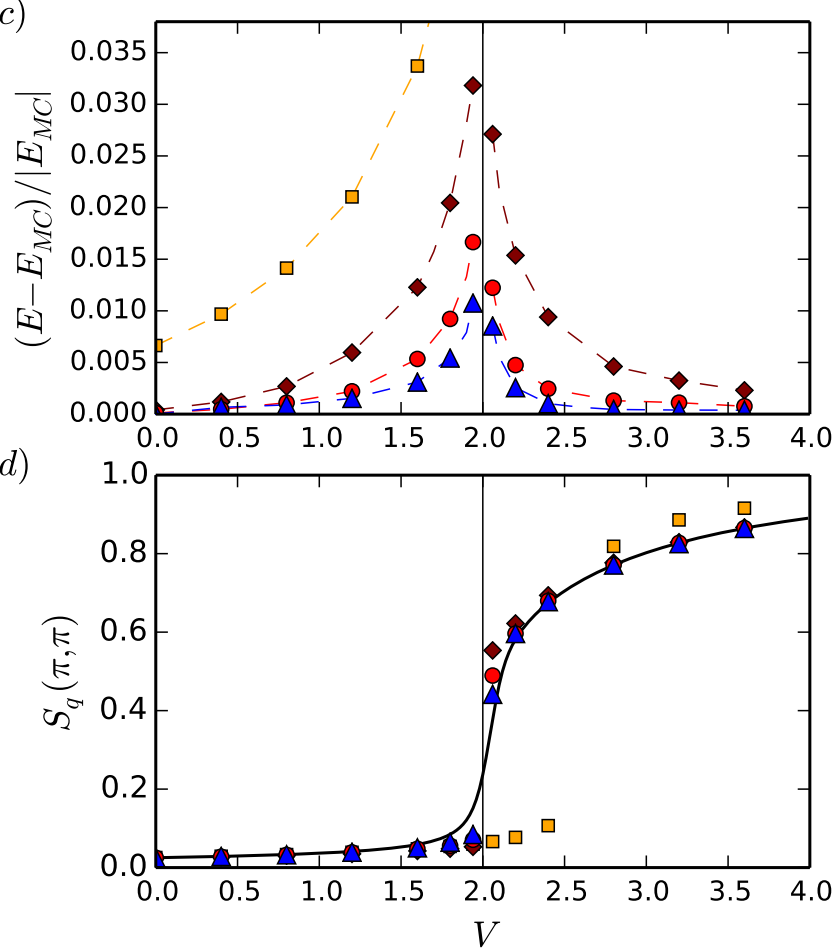

FIG. 3. Results of VMC simulations of hardcore bosons on $4 \times 4$ (left panel) and $8 \times 8$ (right panel) lattice with periodic boundary conditions obtained for different input wave functions: a two-parameter Jastrow wave function (orange squares), a TPS with the bond dimension $D=2$ (maroon diamonds), a projected $D=2$ TPS on the Jastrow factor wave function (red circles), and a TPS with $D=3$ (blue triangles). The upper panels show a relative energy error compared to (a) the ED and (c) the QMC simulations, and the inset shows fidelity obtained for the $4 \times 4$ system. The lower panels display the structure factor $S_{q}(\pi, \pi)$ : (b) the ED and (d) the QMC results are denoted by solid lines.

Here the Jastrow operator is defined as $\hat{J}_{\gamma, \beta} \equiv$ $\exp \left(\gamma \sum_{\langle i j\rangle} n_{i} n_{j}+\beta \sum_{\langle\langle i j\rangle\rangle} n_{i} n_{j}\right)$, where the sum over $\langle i j\rangle$ counts the number of the nearest-neighbor occupied sites and $\langle\langle i j\rangle\rangle$ the number of the next-nearest-neighbor occupied sites. Simulating the Jastrow-type wave function we find that the energy error obtained with respect to the ED results grows rapidly with the value of $V$ and reaches the value of about $20 \%$ in the CDW insulating phase shown in Fig. 3(a).

For the TPS [Eq. (1)], we assume periodic boundary conditions and a bipartite structure for tensor elements: all tensors with the same physical index on a given sublattice ( $A$ or $B$ ) have the same elements, moreover, $\hat{T}_{[i \in A]}^{1(0)}=\hat{T}_{[i \in B]}^{0(1)}$, where the upper (physical) index denotes site occupation. We further assume that the tensor elements are real and symmetric, and that they have rotational symmetry, i.e., the tensor elements are related,

$$
\hat{T}_{i j k l}=\hat{T}_{j k l i}=\hat{T}_{k l i j}=\hat{T}_{l i j k},
$$

where $i, j, k, l=1, \ldots, D$ are the indices for the virtual bonds. We begin by optimizing the TPS with the bond dimension $D=2$. One can see in Fig. 3(a) that indeed the TPS is a much better trial wave function than the simple Jastrow-type wave function for all values of interaction $V$. However, the computational cost to obtain reliable results from the TPS is much higher even for a $4 \times 4$ lattice system and the smallest nontrivial bond dimension $D=2$. As we can expect, the TPS with $D=3$ offers much better description to the ground state, especially around the transition to the CDW insulator. Increasing $D$ is a systematic way of improving the quality of the wave function, but the contraction cost increases even further. Moreover, the number of different tensor parameters that need to be optimized increases (in our case from 12 to 48 ).

We now compare the TPS results to our TPPS [Eq. (3)] approach in which we use a two-parameter Jastrow input wave function. Including the two Jastrow parameters in the TPPS practically does not increase the computational cost with respect to the TPS with $D=2$. Fig. 3 (a) shows that the results in a $4 \times 4$ lattice system look very promising in terms of the energy errors, which are much closer to the results of the TPS with $D=3$, especially where the TPS gives the maximal energy error for $V \approx 3$.

In the inset to Fig. 3(a) we show the overlap (fidelity) 
$F$ between the ED wave function $\left|\Phi_{E D}\right\rangle$ and the wave functions $|\Psi\rangle$ optimized by our simulations for the $4 \times 4$ system.

$$
F=\left|\left\langle\Psi \mid \Phi_{E D}\right\rangle\right|
$$

For small $V$ the fidelity values are close to unity, with the biggest deviation observed for the Jastrow-type wave function. However, at $V \approx 4 t$ there is a jump for both TPS wave functions with $D=2$. The lower fidelity values can be understood as a consequence of the wave function converging to one of the two symmetry-breaking charge ordered states. On the other hand, the Jastrow-type wave function in the form used in this work cannot break the symmetry between the two ordered states, retaining a large overlap with the ED results. As for the $D=3$ TPS wave function, more variational parameters allows for a superposition of the two solutions in the range of $V$ considered here, giving a fidelity value close to one. In Fig. 3(b), we also show the structure factor $S_{q}(\pi, \pi)$ and compare it to the ED results. We notice that all tensornetwork based trial states agree with the exact results very well, while the Jastrow-type wave function shows the biggest difference near the critical point $(V=2)$.

In Fig. 3(c) and (d) a similar analysis is presented for an $8 \times 8$ lattice system, for which we compare the results from the tensor-network projection method to the stochastic series expansion (SSE) QMC simulations. ${ }^{36-38}$ We observe again that the results of the TPS with $D=2$ can be considerably improved by either increasing the bond dimension to $D=3$ or adding the Jastrow factors to the TPPS. The energy errors are very similar for the TPS with $D=3$ and the TPPS with $D=2$. The main difference with respect to the SSE method for all input wave functions we use here is the behavior of these wave functions around the critical point. We find that the VMC simulation in the $2 \mathrm{D} t-V$ model falls into two minima rather than shows a continuous transition. The quantitative differences in the structure factor are the smallest for the $D=3$ TPS, and become more pronounced for $D=2$ TPPS and $D=2$ TPS, especially close to $V=2$.

\section{B. Spinless fermions}

We consider the spinless fermionic $t$ - $V$ model at half filling whose Hamiltonian can be expressed in the same form as that in Eq. (8), but now the creation/annihilation operators are fermionic. It is one of the simplest Hamiltonians for simulating interacting fermions, and it has been discussed as a model capturing the essential physics of several organic materials (at one-quarter filling). ${ }^{39}$ The phase diagram of the $2 \mathrm{D} t-V$ model at half filling was first studied by Gubernatis et al. using several techniques, including finite-temperature determinantal QMC. ${ }^{40}$ The random-phase approximation calculation of the temperature of the CDW transition for small $V$ gives $T_{C D W} \sim \exp (-\pi / \sqrt{V})$, indicating that the CDW phase
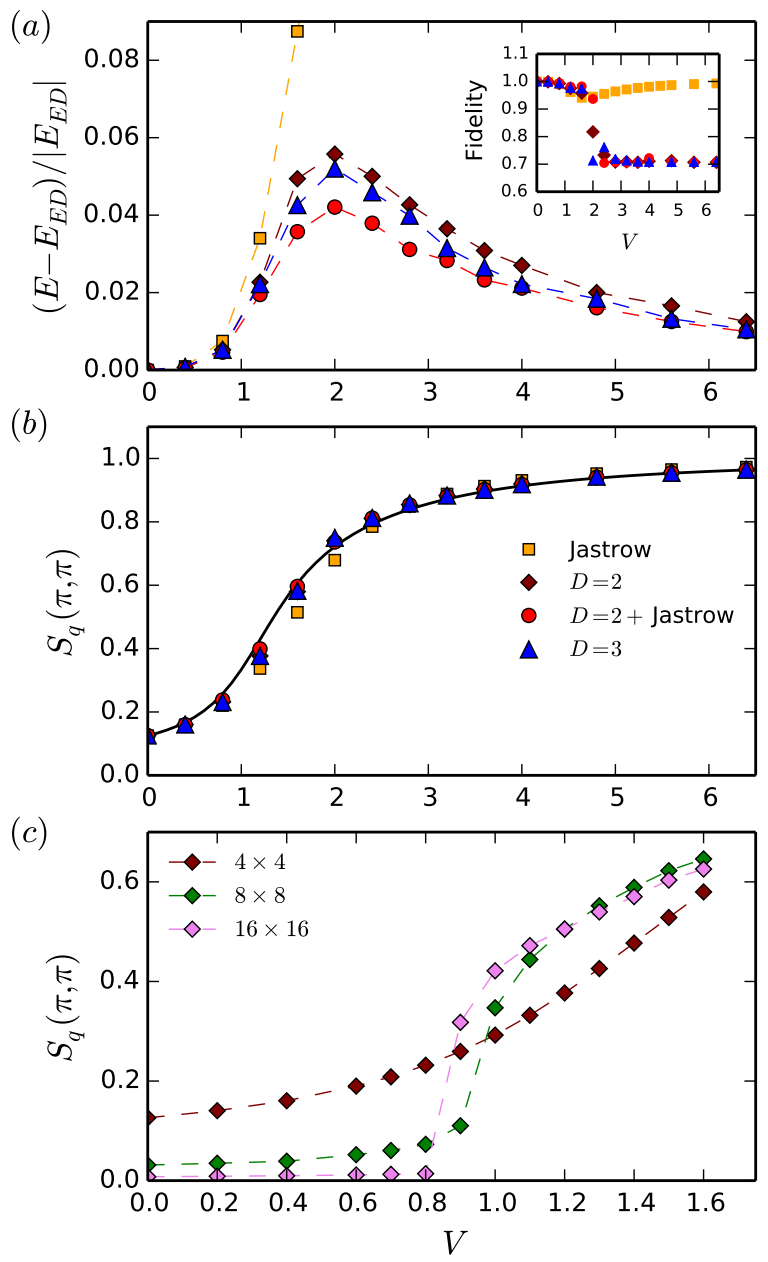

FIG. 4. Results of VMC simulations of the spinless fermion model. Panels (a,b) show data for a $4 \times 4$ lattice system: (a) the relative energy error and (inset) the fidelity; (b) the structure factor $S_{q}(\pi, \pi)$. All input wave functions include the Slater determinant: a two-parameter Jastrow-type wave function (orange squares), a TPPS with the bond dimension $D=2$ (maroon diamonds), a $D=2$ TPPS with the Jastrow factors (red circles) and a TPPS with $D=3$ (blue triangles). The ED data for $S_{q}(\pi, \pi)$ are denoted by a solid line. (c) The structure factor $S_{q}(\pi, \pi)$ for a series of $2^{n} \times 2^{n}$ clusters $(n=$ $2,3,4)$ calculated by using a TPPS with the bond dimension $D=2$.

is the ground state for all positive values of $V$. However, the numerical results of their work do not reach the weak coupling regime.

The model has been also considered as a particular case in the field theory study of Hubbard-like lattice models, confirming that there is no metallic phase in two dimensions. ${ }^{41}$ Recently, a contradictory result has been obtained by simulating so called string-bond states. ${ }^{42}$ The finite size scaling of the structure factor is consistent with a nonzero $V_{c}$ and the value estimated for the critical point $V_{c}$ is $0.45 \pm 0.02$. 
In the previous study of the 1D spinless fermion model, using a matrix-product projected state with a noninteracting fermionic state as an input wave function, significant improvements to the ground-state energy and correlations have been found with respect to regular MPS. ${ }^{31}$ These findings motivate us to propose a TPPS ground state with the SL as an input wave function, which is the ground state for $V=0$. It can be easily expressed as a product state in momentum space:

$$
\left|\Psi_{S L}\right\rangle=\prod_{k<k_{F}} c_{k}^{\dagger}|0\rangle
$$

where $k_{F}$ denotes the Fermi momentum. We apply antiperiodic boundary condition in the $y$ direction in order to avoid degeneracies at the Fermi level which occur in the case of periodic boundary conditions for a series of $2^{n} \times 2^{n}$ clusters $(n=1,2, \ldots)$. Note that it also changes the symmetry of the tensor network: instead of rotational symmetry used for the bosonic system, we impose here $x$ and $y$ reflectional symmetry for the elements of the tensors.

We also use more general input states including a Jastrow-type wave function,

$$
\left|\Psi_{S L+J}\right\rangle=\hat{J}_{\gamma, \beta}\left|\Psi_{S L}\right\rangle .
$$

The Fourier transform of the SL wave function to the site representation leads to Slater determinants as coefficients in front of the configuration vectors $|\alpha\rangle$ :

$$
\left|\Psi_{S L}\right\rangle=\sum_{\alpha}\left\langle\alpha \mid \Psi_{S L}\right\rangle|\alpha\rangle
$$

In Fig. 4(a) we present the energy error for SL TPPS for $D=2$ and 3 with ED results $(4 \times 4$ system $)$. First of all, the SL TPPS is exact at $V=0$ and remains a good approximation of the ground-state wave function for small values of $V$. Unlike in the bosonic case, we do not observe a significant improvement of the energy with the increasing bond dimension. We also consider the $D=2$ TPPS with both Slater determinant and Jastrow factors. As mentioned before, adding Jastrow factors practically does not increase the computational cost, however, the energy error is reduced with respect to the TPPS using the Slater determinant only [cf. red circles and maroon diamonds in Fig. 4(a)]. The fidelity results shown in the inset to Fig. 4(a) start from unity for $V=0$ and then the overlap with the ED wave function slowly decreases as the interaction strength increases until $V \approx 2$. Above this value all TPPS wave functions fluctuate around one of the charge ordered states and the fidelity values are much lower. In Fig. 4(b) we show the structure factor which is in good agreement with ED results.

In order to study the phase transition to the CDW insulating phase, we perform the VMC simulations for larger cluster sizes. We consider the $D=2$ TPPS with Slater determinant only, and examine the size dependence of the jump of the structure factor. In Fig. 4(c), we show the results for the structure factor $S_{q}(\pi, \pi)$ computed for a series of clusters $2^{n} \times 2^{n}$. We observe a significant shift with increasing system size, and thus a careful finite size scaling analysis is necessary which we defer to a future work. We note that adding more correlations into the Jastrow type input wave function might significantly improve the description of the spinless fermion model. ${ }^{27}$

\section{Spinful fermions}

We compare our method to ED data for a spinful fermionic Hubbard model on a $4 \times 4$ cluster at half-filling with (anti-)periodic boundary conditions along the $x(y)$ direction. The fermionic Hubbard Hamiltonian is given by

$$
H_{t U}=-t \sum_{\langle i, j\rangle, \sigma}\left(c_{i \sigma}^{\dagger} c_{j \sigma}+H . c .\right)+U \sum_{i} n_{i \uparrow} n_{i \downarrow},
$$

where $n_{i \sigma}=c_{i \sigma}^{\dagger} c_{i \sigma}$ with spin $\sigma$.

We compare a number of different mean field wave functions as input functions for our method, namely the Slater determinant of spinful fermions (SL), d-BCS and a spin-density wave (SDW). Details about these standard mean-field wave functions can be found in Refs. 43 and 44. We first optimize the unprojected wave-functions and then use the TPPS approach with increasing bond dimension to further optimize the state. We assume the tensors to be real and without any symmetry. For our benchmark, we measure the energy and the anti-ferromagnetic (AF) order parameter - the staggered magnetization, defined as

$$
\langle|M|\rangle=\left\langle\left|\frac{1}{N} \sum_{i} S_{i}^{z} e^{i \mathbf{Q} \cdot \mathbf{R}_{i}}\right|\right\rangle,
$$

where $\mathbf{Q}=(\pi, \pi)$ is the AF ordering vector, $\mathbf{R}_{i}$ is the position of the fermion at site $i$, and $S_{i}^{z}=\frac{1}{2}\left(n_{i \uparrow}-n_{i \downarrow}\right)$. We show in Fig. 5 that the tensors of the TPPS significantly improve the variational energy for all input states $\left|\Psi_{0}\right\rangle$. In the case of the smallest bond dimension $D=1$, the $T_{[i]}^{j_{i}}$ are simply scalars for each $j_{i}=0, \uparrow, \downarrow, \uparrow \downarrow$ and thus the resulting projectors resemble onsite Gutzwiller-type projectors.

In the regime of small $U / t$ shown in Fig. 5(a) and (c), the tensor network, acting on these mean-field states attempt to capture a weak staggered magnetization as $D$ increases. The SL and BCS states show slightly lower energies than the SDW state for any finite $D$. This result implies that the AF correlation built in the SDW wave function is too large and the projectors need to correct for that. In other words, we have to use a large bond dimension of the tensors to project out the AF order in the SDW input state. Choosing a good input wave function thus not only takes care of the fermionic sign, but it efficiently encodes the correlations between fermions which would require a larger bond dimension in regular TPS. 

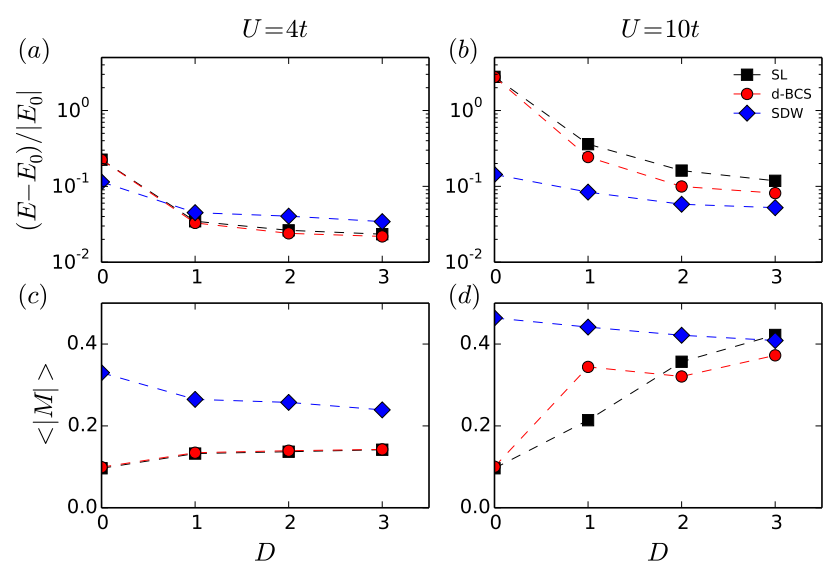

FIG. 5. Relative energy error of $4 \times 4$ spinful fermions in the Hubbard model as a function of the bond dimension $D$ at (a) $U / t=4$ and (b) $U / t=10$ for different input states $\left|\Psi_{0}\right\rangle:$ SL, $d$-BCS and SDW. The ED data are taken from Ref. 45. The lower panels show the staggered magnetization vs $D$ at (c) $U / t=4$ and (d) $U / t=10$. The $D=0$ values correspond to the unprojected input wave functions.

In the limit of large $U / t$ we expect the ground state to form AF order. We find in Fig. 5(b) indeed that the unprojected input wave function of the SDW state is much closer to the ground-state than the unprojected SL and BCS states. The tensor-product projectors, acting on both SL and BCS input states, exhibits a staggered magnetization as shown in Fig. 5(d). In particular, already the $D=1$ tensors substantially lower the energy of the input states. We furthermore find that the SDW wave function has the lowest energy for all $D$ as the input wave-function already takes care of the AF correlation. In order to approach the same energy level as the SDW state, the TPPS with SL or BCS state need larger bond dimensions. This demonstrates that TPPS are a promising tool to represent ground states of 2D Hubbard models.

\section{CONCLUSIONS}

We investigated a numerical method based on tensorproduct projected states (TPPS), which combine re- cently developed tensor-network based states and conventional trial wave functions, to study the ground state properties of quantum many-body systems. Using conventional VMC methods, we demonstrated the applicability and flexibility of TPPS in describing the ground state energy and correlations for several quantum systems. Choosing a suitable input state, which captures some known features of the ground state, we were able to reach significantly lower energy than using a standard TPS with the same bond dimension. Thus the numerical effort to search the exact ground state can be considerably reduced, both by lowering the cost of tensor contraction and the number of parameters that need to be optimized. Our results suggest that the approach would be useful in simulating fermionic systems where quantum Monte Carlo simulations face the sign problem, and in the case where the TPS has been used but is not currently capable of reaching sufficient accuracies. Furthermore, a proper quantum-number projection and using symmetric tensors to filter out excited states with other quantum numbers may potentially further improve the variational energy of the TPPS. Finally, we note that TPPS's are capable of expressing states with non-trivial topological order ${ }^{46}$ as there are no constraints on the input wave functions.

\section{ACKNOWLEDGEMENTS}

This work is partly supported by the Chinese Academy of Engineering Physics and Ministry of Science and Technology (CPC), and the Ministry of Science and Technology in Taiwan under Grants No. 100-2112-M-002013-MY3 (YJK, HWC, OS), 100-2923-M-004-002-MY3 (YJK), and 102-2112-M-002-003-MY3 (YJK). YJK acknowledges travel support from NCTS in Taiwan. OS gratefully acknowledges support from the visitors program of MPI-PKS Dresden. We also acknowledge the hospitality of the Benasque Center for Science where part of this work was done.
* cpc63078@gmail.com

$\dagger$ frankp@pks.mpg.de

¥ yjkao@phys.ntu.edu.tw

1 X.-G. Wen, Quantum Field Theory of Many-Body Systems (Oxford University Press, Oxford, 2004).

2 L. Balents, Nature 464, 199 (2010).

3 S. R. White, Phys. Rev. Lett. 69, 2863 (1992).

${ }^{4}$ W. von der Linden, Phys. Rep. 220, 53 (1992).
${ }^{5}$ S. Östlund and S. Rommer, Phys. Rev. Lett. 75, 3537 (1995)

${ }^{6}$ F. Verstraete and J. I. Cirac, arXiv:cond-mat/0407066.

7 F. Verstraete and J. I. Cirac, Phys. Rev. A 70, 060302 (2004).

8 Y.-Y. Shi, L.-M. Duan, and G. Vidal, Phys. Rev. A 74, 022320 (2006).

9 N. Schuch, M. M. Wolf, F. Verstraete, and J. I. Cirac, Phys. Rev. Lett. 100, 040501 (2008). 
10 A. W. Sandvik, Phys. Rev. Lett. 101, 140603 (2008).

11 V. Murg, F. Verstraete, Ö. Legeza, and R. M. Noack, Phys. Rev. B 82, 205105 (2010).

12 F. Mezzacapo, N. Schuch, M. Boninsegni, and J. I. Cirac, New J. Phys. 11, 083026 (2009).

13 H. J. Changlani, J. M. Kinder, C. J. Umrigar, and G. K.-L. Chan, Phys. Rev. B 80, 245116 (2009).

14 R. Hübener et al., Phys. Rev. A 79, 022317 (2009); R. Hübener et al., Phys. Rev. B 84, 125103 (2011).

15 R. Hübener, V. Nebendahl, and W. Dür, New J. Phys. 12, 025004 (2010).

16 V. Murg, F. Verstraete, and J. I. Cirac, Phys. Rev. A 75, 033605 (2007).

17 V. Murg, F. Verstraete, and J. I. Cirac, Phys. Rev. B 79, 195119 (2009).

18 B. Bauer, G. Vidal, and M. Troyer, J. Stat. Mech.: Theory Exp. P09006 (2009).

19 L.Wang, I. Pižorn, and F. Verstraete, Phys. Rev. B 83, 134421 (2011).

${ }^{20}$ G. Vidal, Phys. Rev. Lett. 99, 220405 (2007).

21 Z.-C. Gu, M. Levin, and X.-G. Wen, Phys. Rev. B 78, 205116 (2008).

${ }^{22}$ H. C. Jiang, Z. Y. Weng, and T. Xiang, Phys. Rev. Lett. 101, 090603 (2008).

${ }^{23}$ L. Wang, Y.-J. Kao, and A. W. Sandvik, Phys. Rev. E 83, 056703 (2011); J.-F. Yu and Y.-J. Kao, Phys. Rev. B 85, 094407 (2012).

24 Z.-C. Gu, Phys. Rev. B 88, 115139 (2013).

25 S. Wouters, B. Verstichel, D. Van Neck, and G. K.-L. Chan, Phys. Rev. B 90, 045104 (2014).

${ }^{26}$ B. K. Clark and H. J. Changlani, arXiv:1404.2296.

27 E. Neuscamman H. Changlani, J. Kinder, and G. K.-L. Chan, Phys. Rev. B 84, 205132 (2011).
28 E. Neuscamman, C. J. Umrigar, and G. K.-L. Chan, Phys. Rev. B 85, 045103 (2012).

29 Ö. Legeza and J. Sólyom Phys. Rev. B 68, 195116 (2003).

30 G. Barcza, Ö. Legeza, K. H. Marti and M. Reiher, Phys. Rev. A 83, 012508 (2011).

31 C.-P. Chou, F. Pollmann and T.-K. Lee, Phys. Rev. B 86, 041105(R) (2012).

32 S. Sorella, Phys. Rev. B 64, 024512 (2001).

33 S. Yunoki and S. Sorella, Phys. Rev. B 74, 014408 (2006).

34 M. Capello, Variational description of Mott insulators, PhD thesis, SISSA (2006).

${ }^{35}$ F. Hébert, G.G. Batrouni, R.T. Scalettar, G. Schmid, M. Troyer and A. Dorneich, Phys. Rev. B 65, 014513 (2001).

36 A. W. Sandvik and J. Kurkijärvi, Phys. Rev. B 43, 5950 (1991).

37 A. W. Sandvik Phys. Rev. B 59, R14157(R) (1999).

38 O. F. Syljuåsen and A. W. Sandvik Phys. Rev. E 66, 046701 (2002).

39 R. H. McKenzie, J. Merino, J. B. Marston, and O. P. Sushkov, Phys. Rev. B 64, 085109 (2001).

40 J. E. Gubernatis, D. J. Scalapino, R. L. Sugar, and W. D.Toussaint, Phys. Rev. B 32, 103 (1985).

41 M. S. Foster and A. W. W. Ludwig, Phys. Rev. B 77, 165108 (2008)

42 J. -P. Song and R.T. Clay, Phys. Rev. B 89, 075101 (2014).

43 C. Gros, Ann. Phys. 189, 53 (1989).

44 M. Ogata and H. Fukuyama, Rep. Prog. Phys. 71, 036501 (2008).

45 D. Tahara and M. Imada, J. Phys. Soc. Jpn. 77, 114701 (2008).

46 T. B. Wahl, H. H. Tu, N. Schuch, and J. Cirac, Phys. Rev. Lett., 111, 236805 (2013) 